\title{
CONTROL SYSTEM DESIGN OF A TRAVELING CRANE USING $\mathrm{H}_{\infty}$ CONTROL THEORY
}

\author{
Nariyuki Kodani*, Shigeto Ouchi* and Yuji Todaka** \\ *Department of Electrical Engineering, Tokai University, ouchis@keyaki.cc.u-tokai.ac.jp \\ **Fuji Electric Co., Ltd.
}

\begin{abstract}
For a traveling crane, various control methods such as fuzzy control and optimum control are studied. In the control of an actual traveling crane, it is important to accurately stop the crane at the desired position by positioning control and anti-sway control. In this pare, we used the $\mathrm{H}_{\infty}$ control theory which performs well even when there are modeling errors and parameter variations. The results of several experiments confirmed that the $\mathrm{H}_{\infty}$ control system is effective.
\end{abstract}

\section{INTRODUCTION}

There are several methods of controlling the sway of traveling cranes, such as fuzzy control ${ }^{|1|}$ and optimal control ${ }^{|2|}$, each of which is reported to be effective ${ }^{[3]}$. The development of a simple method for designing control systems is important for field engineers. In a control system, the controller is generally designed by using a mathematical model of the controlled object. However, it is impossible to make a model which accurately expresses the characteristics of the controlled object. Therefore, the controller obtained by this method is not always appropriate for actual systems, even if it is valid theoretically.

In this paper, we used the $\mathrm{H}_{\infty}$ control theory which is a robust control technique for modeling errors and parameter variations, and confirmed its effectiveness by simulations and experiments. ${ }^{[5]}$

\section{CONTROLLED OBJECT}

Figure 1 is a model of a controlled object, where $x$ is the position of the trolley, $l$ the rope length, $m$ the mass of load, $M$ the mass of trolley, $\theta$ the sway angle of load, and $u$ the external force. For the model shown in Fig. 1, the equation of motion for the trolley can be expressed by

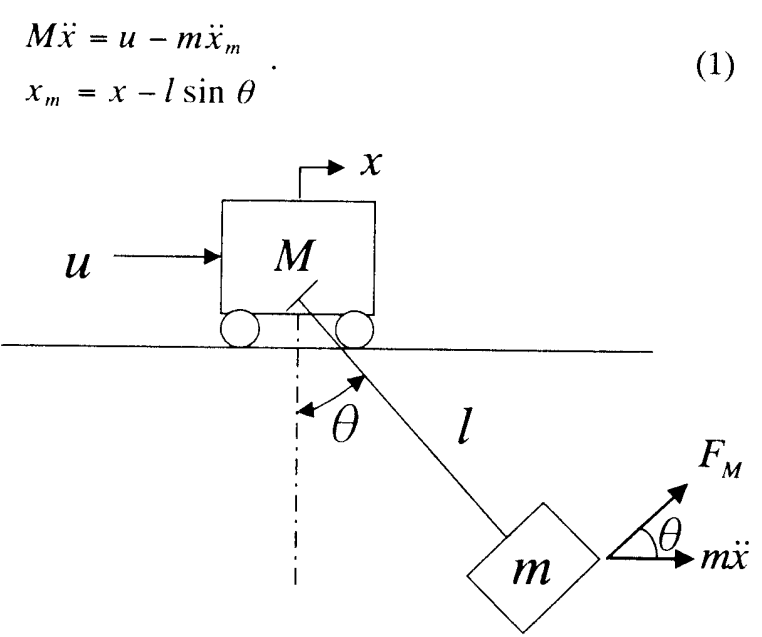

Fig.1 Crane model

Also, the equation of motion relating to the load can be expressed by

$$
\begin{aligned}
& m l \ddot{\theta}=-m g \sin \theta+F_{M} \\
& F_{M}=m \ddot{x} \cos \theta
\end{aligned}
$$

From equations (1) and (2), the equations of motion of a traveling crane are

$$
\begin{aligned}
& (M+m) \ddot{x}+m l \dot{\theta}^{2} \sin \theta=m l \ddot{\theta} \cos \theta+u \\
& l \ddot{\theta}+g \sin \theta=\ddot{x} \cos \theta
\end{aligned}
$$

Upon approximating these equations around $\theta=0$ and assuming $M \gg m$, equation (3) is transformed into the following equation.

$$
\begin{aligned}
& l \ddot{\theta}+g \theta=\ddot{x} \\
& \ddot{x}=\frac{1}{M} u
\end{aligned}
$$

From the above equation, the state-space equation of a traveling crane can be expressed by

$$
\begin{aligned}
& \dot{X}=A_{P} X+B_{P} u \\
& y=C_{P} X,
\end{aligned}
$$

and supposing that the state variable is 


$$
X=\left\lfloor\begin{array}{llll}
x & \dot{x} & \theta & \dot{\theta}
\end{array}\right]^{T}
$$

where,

$$
\begin{aligned}
A_{P} & =\left[\begin{array}{llll}
0 & 1 & 0 & 0 \\
0 & 0 & 0 & 0 \\
0 & 0 & 0 & 1 \\
0 & 0 & -g / 1 & 0
\end{array}\right] B_{P}=\left[\begin{array}{c}
0 \\
1 / M \\
0 \\
1 / M l
\end{array}\right] \\
C_{P} & =\left[\begin{array}{llll}
1 & 0 & 0 & 0 \\
0 & 1 & 0 & 0 \\
0 & 0 & 1 & 0 \\
0 & 0 & 0 & 1
\end{array}\right]
\end{aligned}
$$$$
M=18[\mathrm{~kg}], g=9.8\left[\mathrm{~m} / \mathrm{s}^{2}\right] \text { and } l=1[\mathrm{~m}] \text {. }
$$

\section{3. $\mathrm{H}_{\infty}$ CONTROL SYSTEM DESIGN}

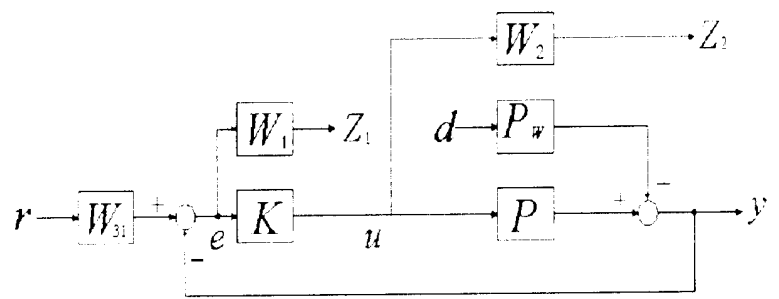

Fig.2 Block diagram

Let us consider the system shown in Fig. 2, in order to design a control system which follows a reference input and is robust against disturbances and parameter variations.

In Fig. 2, $\mathrm{P}$ is the plant, $\mathrm{K}$ the controller, $\mathrm{W}_{\mathrm{t}}, \mathrm{W}_{2}, \mathrm{~W}_{31}$ and $P_{w}$ the weighting functions, $r$ the control input, $d$ the disturbance, $y$ the measured output, and $z_{1}, z_{2}$ the controlled outputs.

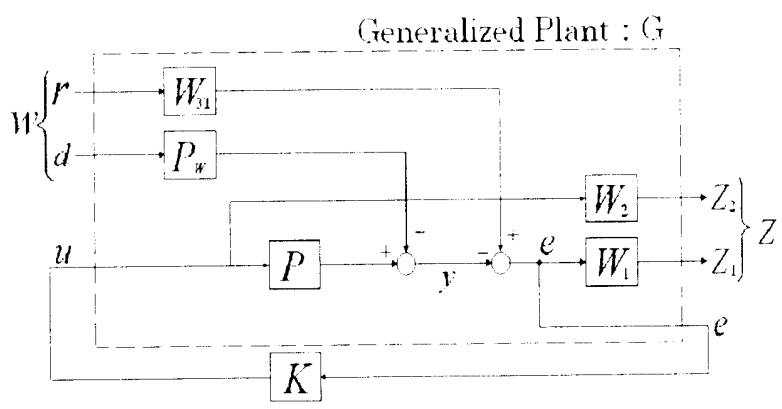

Fig.3 Block diagram of the $H_{\infty}$ control

From Fig. 2, the $\mathrm{H}_{\infty}$ control system can be expressed as shown in Fig. 3. From Fig. 3, the generalized plant $G$ including the weights can be expressed by

$$
G=\left[\begin{array}{ccc}
W_{1} W_{31} & W_{1} P_{w} & -W_{1} P \\
0 & 0 & W_{2} \\
W_{31} & P_{w} & -P
\end{array}\right]
$$

and the relation between input and output is

$$
\left[\begin{array}{l}
z_{1} \\
z_{2} \\
e
\end{array}\right]=G\left[\begin{array}{l}
r \\
d \\
u
\end{array}\right]
$$

If plant $P$ and weights $W_{1}, W_{2}, W_{31}$ and $P_{w}$ are given by

$$
\begin{aligned}
& W_{1}=\left[\begin{array}{l|l}
A_{w 1} & B_{w 1} \\
\hline C_{w 11} & D_{w 1}
\end{array}\right] \quad W_{2}=\left[\begin{array}{l|l}
A_{w 2} & B_{w 2} \\
\hline C_{w: 2} & D_{w 2}
\end{array}\right] \\
& W_{31}=\left[\begin{array}{l|l}
A_{p} & B_{w 311} \\
\hline C_{p} & D_{w \cdot 31}
\end{array}\right] \quad P_{w}=\left[\begin{array}{l|l}
A_{P} & B_{w 32} \\
\hline C_{p} & D_{w 32}
\end{array}\right] \\
& P=\left[\begin{array}{c|c}
A_{p} & B_{p} \\
\hline C_{p} & 0
\end{array}\right] .
\end{aligned}
$$

Then we obtain the state-space equation as

$$
\begin{aligned}
G & =\left[\begin{array}{ccc|cc:c}
A_{w 1} & B_{w 1} C_{p} & 0 & B_{w 1} D_{w 31} & B_{w 1} D_{w 32} & 0 \\
0 & A_{p} & 0 & B_{w 31} & B_{w 32} & -B_{p} \\
0 & 0 & A_{w 2} & 0 & 0 & B_{w 2} \\
\hline C_{w 1} & D_{w 1} C_{p} & 0 & D_{w 1} D_{w 31} & D_{w 1} D_{w 32} & 0 \\
0 & 0 & C_{w 2} & 0 & 0 & D_{w 2} \\
\hdashline 0 & C_{p} & 0 & D_{w 31} & D_{w 32} & 0
\end{array}\right] \\
& =\left[\begin{array}{c|cc}
A & B_{1} & B_{2} \\
\hline C_{1} & D_{11} & D_{12} \\
C_{2} & D_{21} & D_{22}
\end{array}\right] .
\end{aligned}
$$

Given equation (8) is by

$$
\begin{aligned}
& {\left[\begin{array}{l}
z \\
e
\end{array}\right]=G\left[\begin{array}{l}
w \\
u
\end{array}\right]=\left[\begin{array}{ll}
G_{11} & G_{12} \\
G_{21} & G_{22}
\end{array}\right]\left[\begin{array}{l}
w \\
u
\end{array}\right]} \\
& {\left[\begin{array}{ll}
z_{1} & z_{2}
\end{array}\right]^{T}=z,\left[\begin{array}{ll}
r & d
\end{array}\right]^{T}=w}
\end{aligned}
$$

we let the control law be

$$
u=K e \text {. }
$$

Then transfer function $\Phi$ from w to z can be expressed by

$$
z=\Phi w=\left\{G_{11}+G_{12} K\left(I-G_{22} K\right)^{-1} G_{21}\right\} \text {. }
$$

As is well known ${ }^{[4]}, \mathrm{H}_{\infty}$ control theory can be used to obtain the controller $\mathrm{K}$ which satisfies the following conditions:

1) $\Phi$ is internally stable, and

2) $\|\Phi\|_{x}<\gamma$ ( $\gamma$ : Given positive number), on the assumptions that

a1) $\left(\begin{array}{ll}A & B_{2}\end{array}\right)$ can be stabilized and $\left(\begin{array}{ll}C_{2} & A\end{array}\right)$ is detectable,

a2) $D_{12}$ is column full rank and $D_{21}$ is row full rank, and

a3) $G_{12}$ and $G_{21}$ have no invariant zeroes on the imaginary axis. 


\subsection{SELECTION OF WEIGHTS}

For the weighting function $\mathrm{W}$, we considered that $\mathrm{W}_{1}$ is a weight related to steady-state characteristics and quick response, $W_{2}$ a weight related to stability, $W_{31}$ a weight related to a reference input, and $P_{w}$ a weight related to disturbances and we chose the following weights accordingly:

- $\mathrm{W}_{1}$ is determined so as to satisfy the quick response. Particularly for $W_{11}$, a pseudo integration is used so that the trolley position $x$ can follow the reference value.

$$
w_{1}=\left[\begin{array}{cccc}
\frac{0.001}{s+10^{-6}}+30 & 0 & 30 & 0 \\
0 & 38 & 0 & 0 \\
0 & 0 & 20 & 0 \\
0 & 0 & 0 & 1
\end{array}\right] \text {. }
$$

- For $\mathrm{W}_{2}, \mathrm{k}_{\mathrm{m}}$ is determined so as to restrict the control input, and $l_{\mathrm{f}}$ and $h_{\mathrm{f}}$ are determined so as not to be affected by noise. Consequently $\mathrm{W}_{2}$ is as follows:

$$
w_{2}=\frac{s+l_{f}}{s+h_{f}} k_{m} \quad l_{f}=30 \quad h_{f}=100 \quad k_{m}=50 .
$$

- $\mathrm{W}_{31}$ is chosen by considering the step reference as follows:

$$
B_{w 31}=\left[\begin{array}{llll}
0 & 0 & 0 & 0 \\
0 & 0 & 0 & 0 \\
0 & 0 & 0 & 0 \\
0 & 0 & 0 & 0
\end{array}\right] \quad D_{w 31}=\left[\begin{array}{cccc}
2 & 0 & 0 & 0 \\
0 & 1 & 0 & 0 \\
0 & 0 & 1 & 0 \\
0 & 0 & 0 & 1
\end{array}\right] \text {. }
$$

- For $P_{w}, B_{w 32}$ is determined by considering the disturbance and $D_{w 32}$ is determined by considering the sensor noise:

$$
B_{w 32}=\left[\begin{array}{cc}
0 & 0 \\
0.5 & 0 \\
0 & 0 \\
0 & 1.8
\end{array}\right] \quad D_{w 32}=\left[\begin{array}{cc}
70 & 0 \\
0 & 0 \\
0 & 6 \\
0 & 6.5
\end{array}\right] \text {. }
$$

\section{SIMULATION RESULTS}

Figure 4 shows the simulation results. In Fig. 4, the reference position of $1[\mathrm{~m}]$ was given first, and after 45 seconds an impulse disturbance was given. As a result, the position quickly followed the reference value, and even if a disturbance was added to the system, the error from the reference value was small. Figure 5 shows the experimental results. From the comparison between Figs. 4 and 5, it can be seen that the responses in Fig. 5 for the trolley position $x$, the sway angle $\theta$ and the sway angular velocity $\dot{\theta}$ agree well with those in Fig. 4 , except for the trolley velocity $\dot{x}$. This difference is thought to be caused by stall friction of the experimental system. Figure 6 shows the response of $\theta$ for the non-control case. Comparing $\theta$ in Fig. 5 with that in Fig. 6, it is seen that the control system performs better than the non-control one.
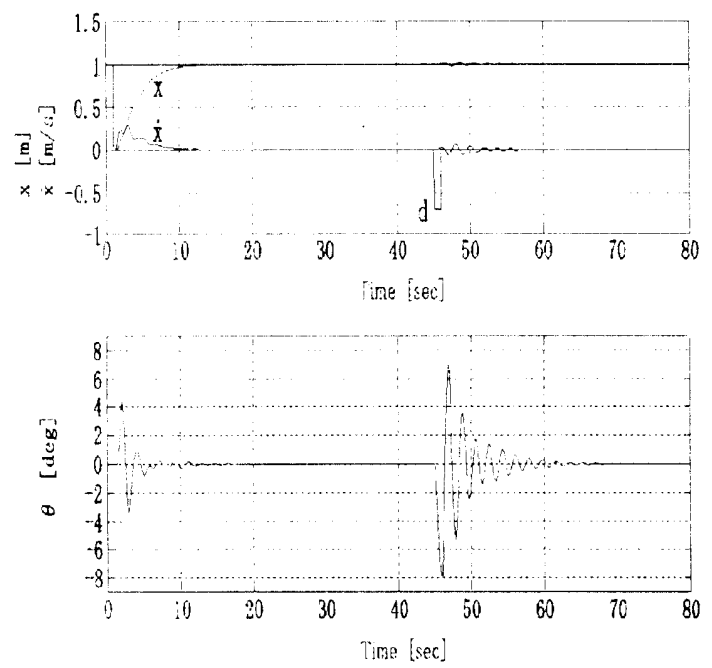

Fig. 4 Simulation
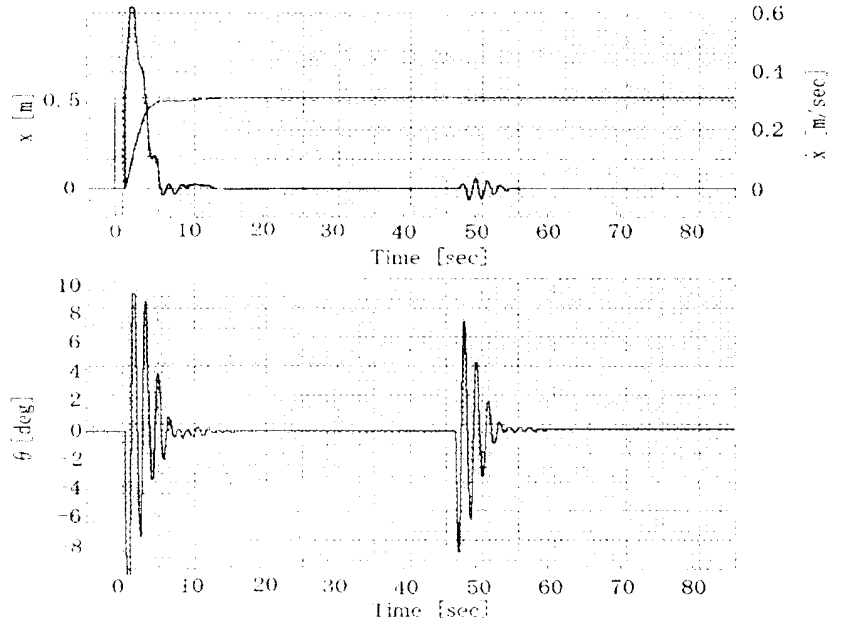

Fig. 5 Experiment

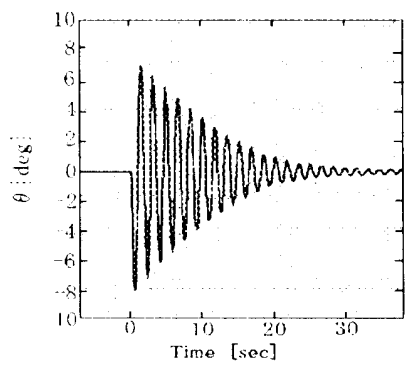

Fig. 6 Response of $\theta$ for non-control 
Figure 7 shows the Bode diagram for the controller. It is seen from Figs. 5 and 6 that the controller for the trolley position $x$ acts as a PID controller, and the controllers for the trolley velocity $\dot{x}$, the sway angle $\theta$ and the sway angular velocity $\dot{\theta}$ act as a PD controller. Consequently, quick response, steady-state error and stability are satisfied for $x$, and it is seen for $\dot{x}, \theta, \dot{\theta}$ that both quick response and stability are satisfied. In particular, the gain characteristics of the controller rise from the resonant frequency of the experimental system. This shows that the controller has a differential characteristic D, and this D serves to reduce the sway of the load.

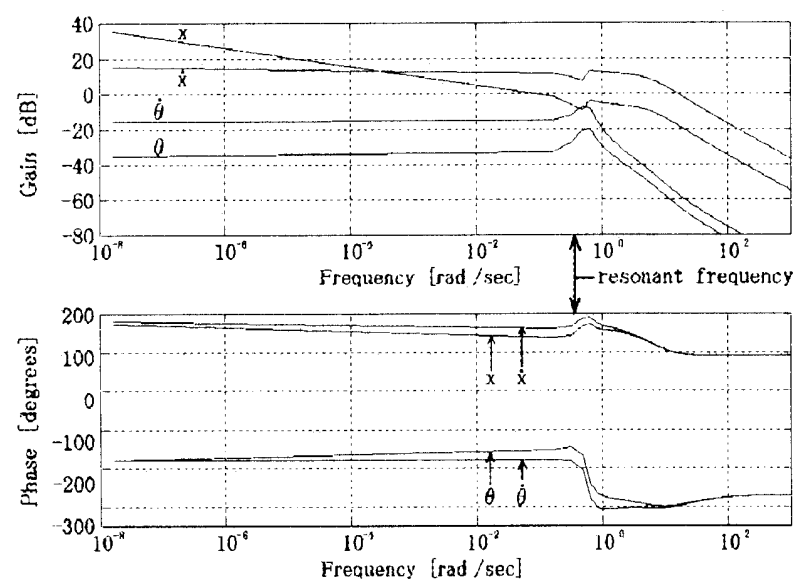

Fig. 7 Bode diagram for the controller

\section{CONCLUSIONS}

For the position and anti-sway control, we obtained good performances in both simulations and experiments. In a future work, we will consider the dead time in the design of the $\mathrm{H}_{\infty}$ control system for traveling cranes.

\section{REFERENCES}

[1] Ito, Migita, Irie. Itou, Nyuui and Maihara "Application of fuzzy control to automatic crane operation" (in Japanese), Journal of Japan Society for Fuzzy Theory and Systems, Vol. 6, No. 2, pp. 402-411, 1994

[2] Yosida, kawaji, Mita and Hara, "Mechanical system control" (in Japanese), Ohmsha, Ltd., 1984

[3] Ouchi, Kikuchi and Todaka, "Anti-sway Control of a Traveling Crane using State Feedback Control" (in Japanese), Proceedings of the 37th SICE Annual Conference, pp. 11-12, 1998

[4] K. Glover and J.C. Doyle, "State-Space Formulas for All Stabilizing Controllers that Satisfy an $\mathrm{H}^{\infty}$-norm Bound and Relation to Risk Sensitivity," Systems \& Control Letters, 11,167, 1988
[5]J.Yanai,M.Murakami,S.Ouchi,K.Z.Liu,Y.Todaka"Anti -Sway Control of a Rotational Crane using the Non-linear Controller"Proceedings of The $6^{\text {th }}$ W.S. on Advanced Motion Control ,pp419-422(2000) 